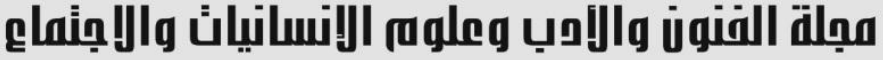

Journal of Arts, Literature, Humanities and Social Sciences

\section{أثر ديكارت في نظرية تشومسكي وتحليله للغة}

\author{
أ.م.د. هلدى صلاح رشيد \\ جامعة تكريت - العراق هلاق
}

الايميل: Dhuda19@yahoo.com

الملخص

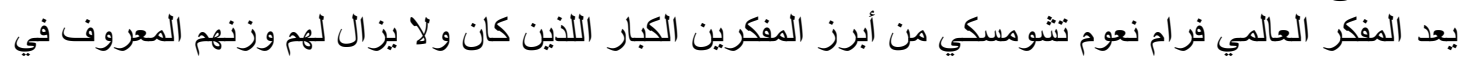

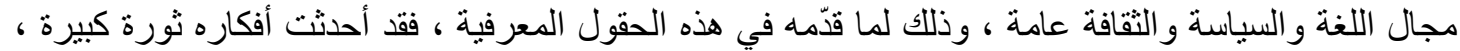
وخاصة في مجال اللغة و النظرة الحديثة للعلوم اللغوية . وقد شكلت هذه الافكار منعطفاً هاماً في هذا المجال المعرفي أثبت بالدليل عجز الانموذج السلوكي التجريبي عن تفسير اللغة.

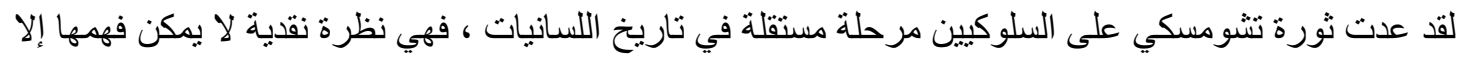

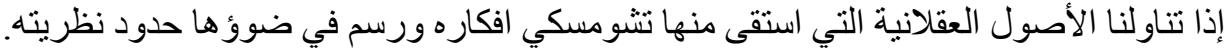

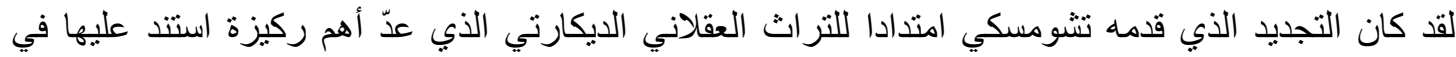

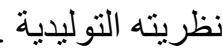
لذا فإنَّ فلسفة ديكارت أثنر و اضح على فئ فكر نتومسكي التوليدي .

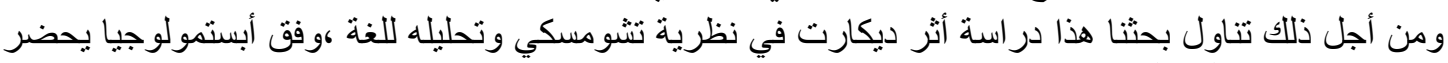

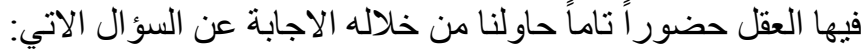

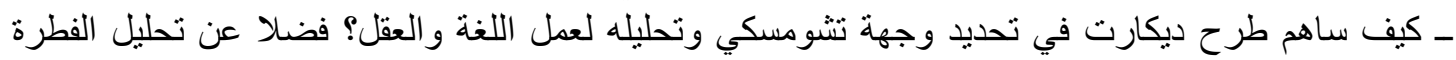
اللغوية بموجب تللك النظرة الثناملة. وقد قسمت البحث على اقسام ليسهل تناوله ، و على النحو الاتي : ـ منطلقات تشو مسكي العقلية.

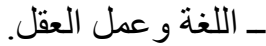

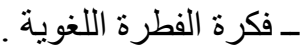




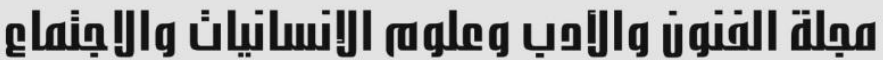

\section{Descartes Influenced Chomsky's Theory and Analysis of Language}

\author{
Assist. Prof. Dr. Huda Salah Rashid \\ Tikrit University - Iraq \\ Email: dhuda19@yahoo.com
}

\begin{abstract}
The international thinker Fram Noam Chomsky is one of the most prominent great thinkers who had and still has a known weight in the field of language, politics and culture in general, for what he presented in these fields of knowledge, as his ideas have revolutionized a great deal, especially in the field of language and the modern view of linguistic sciences.

These ideas constituted an important turning point in this field of knowledge which demonstrated by evidence the inability of the experimental behavioral model to interpret language.

Chomsky's revolution on the behaviorists is considered an independent stage in the history of linguistics. It is a critical view that cannot be understood unless we address the rational origins from which Chomsky drew his ideas and draws the boundaries of his theory.

Chomsky's renewal was an extension of the Cartesian rational heritage, which was the most important pillar on which he built his obstetric theory. So Descartes' philosophy has a clear effect on Chomsky's generative thought.

For this reason, our research deals with the study of Descartes's effect on Chomsky's theory and his analysis of language, according to an epistemology in which the mind attends a complete presence through which we tried to answer the following question: How did Descartes' proposition define Chomsky's view and analysis of language and reason work? As well as analyzing the linguistic instinct according to that comprehensive view.

The research has been divided into sections for easy handling, as follows: Chomsky's mental premises.

Language and the work of the mind.

The idea of language instinct.
\end{abstract}

Keywords: Descartes, Chomsky's Theory, Language analysis. 


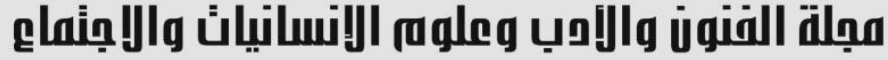

Journal of Arts, Literature, Humanities and Social Sciences

\section{www.jalhss.com}

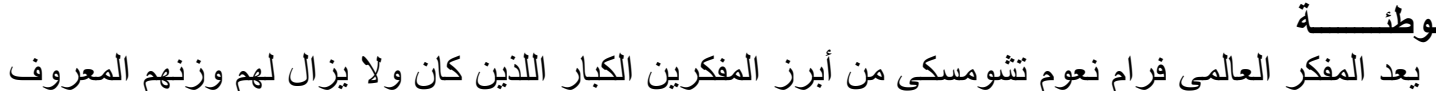

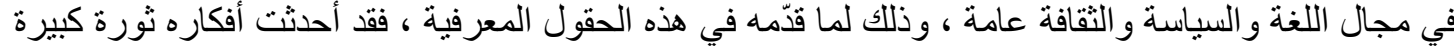

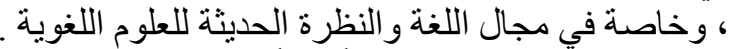
وقد شكلت هذه الافكار منعطفاً هاماً في هذا المجال المعرفي أثبت بالدليل عجز الانموذج السلوكي التجريبي عن

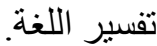

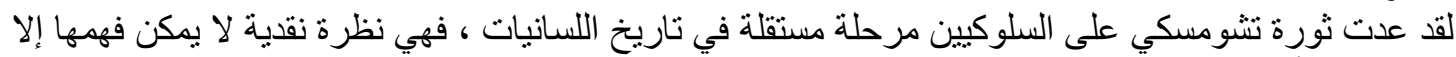

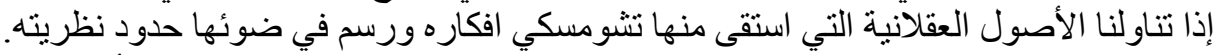

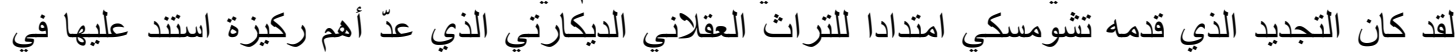
نظريته التوليدية التوانية

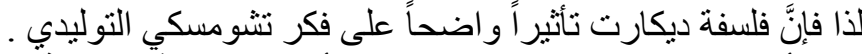

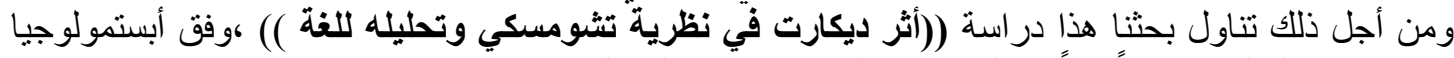

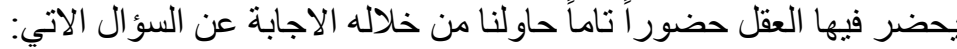

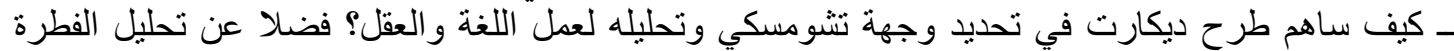
اللغوية بموجب تللك النظرة الثناملة.

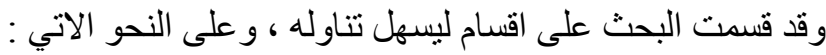
ـ ـ منطلقات تشو مسكي العقلية.

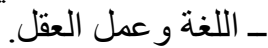

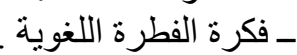

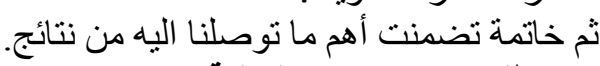

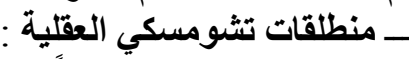

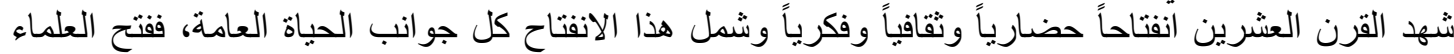

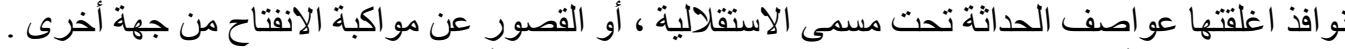

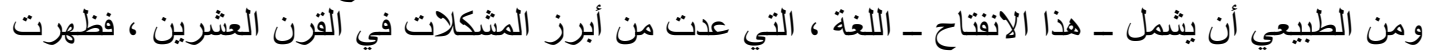

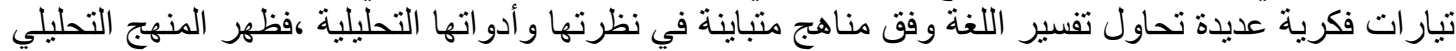

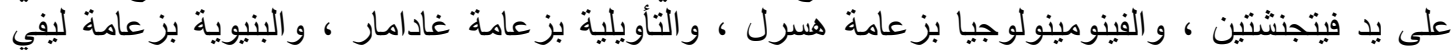

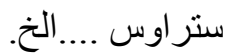

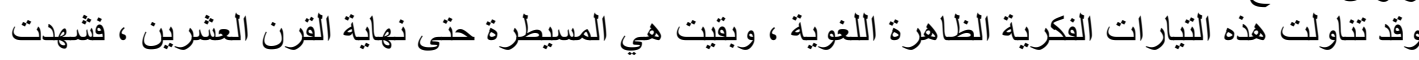

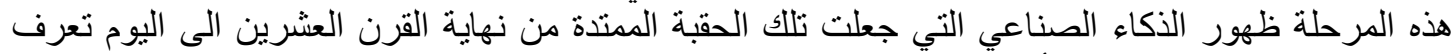

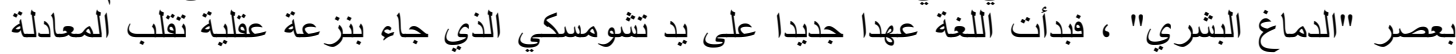

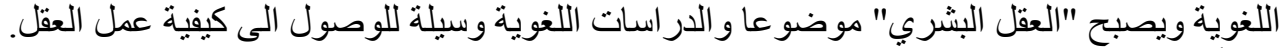

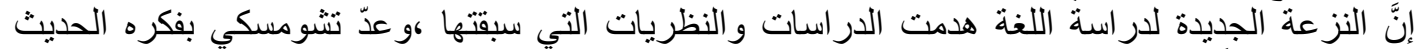
ونظرته للغة رائداً قوض عرش الدراس اسات السلوكية وصدّع أركانها ، فأصبحت عبارة عن أفكار تراثية مضت

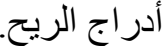
إنَّ نقطة الانطلاق عند تشومسكي تتمنل في نقده للسلوكيين بزعامة بلومفيلد الذي ينظر الى اللغة على أنها لا

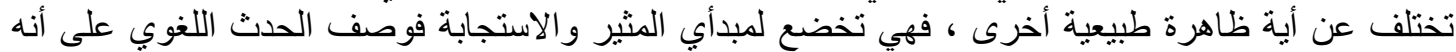

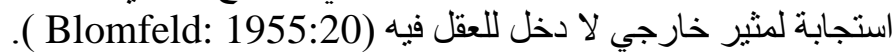

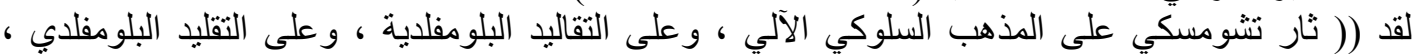

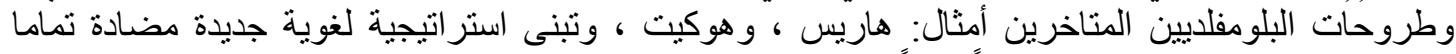

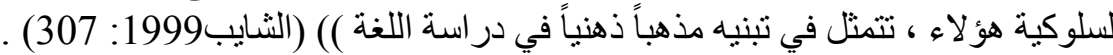

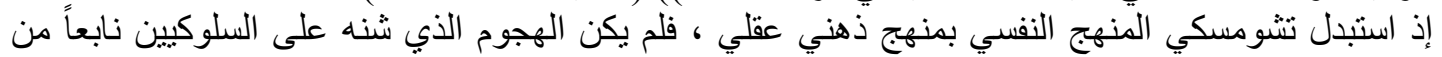

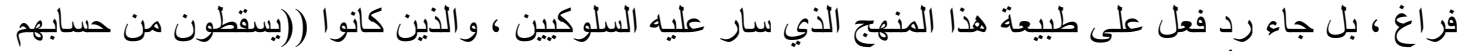
الدور الذي يمكن أن تلعبه البنية الداخلية للكائن الحي نفسه في عمليتي التعلم و السلوك الحقيقي )) (كارلنغ 1998: 


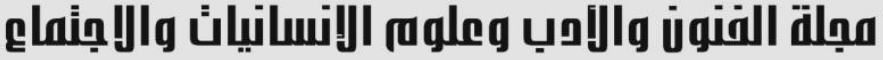

Journal of Arts, Literature, Humanities and Social Sciences www.jalhss.com

12)، لذا ((كان تشومسكي مقتنعا بأنَّ البنية الداخلية للكائن الحي تمثل المفتاح الرئيس و المركزي في فهم السلوك

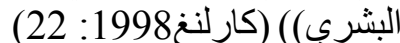

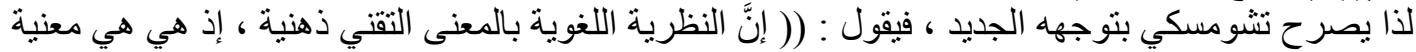

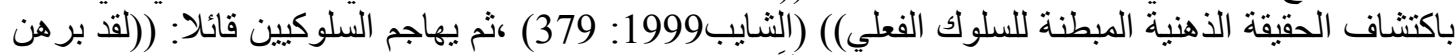
التفكير التجريبي و علم السلوك الذي تطور ضمنهـ ، أنَّهما عقيمان)) (الثايب 1999: 380 380)

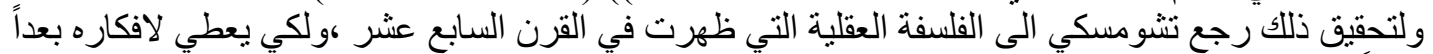

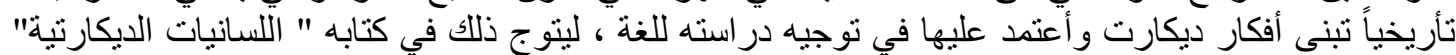

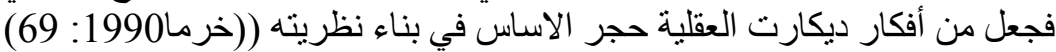

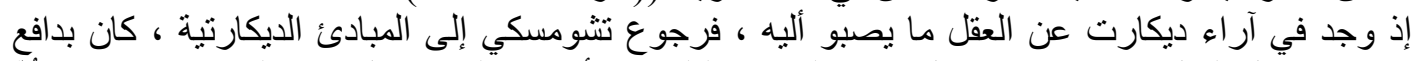

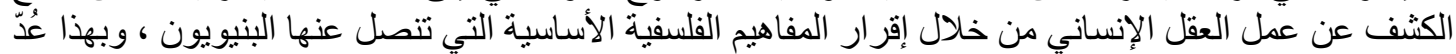

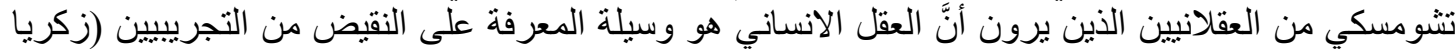

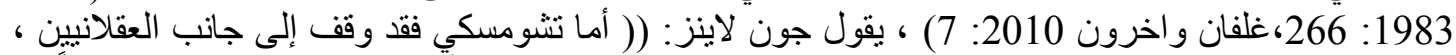

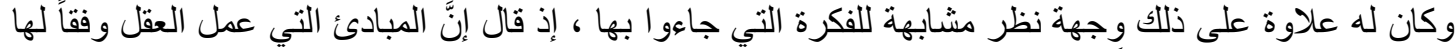

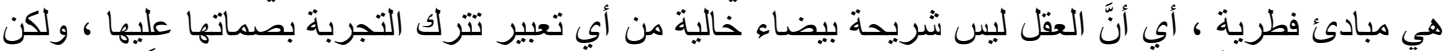

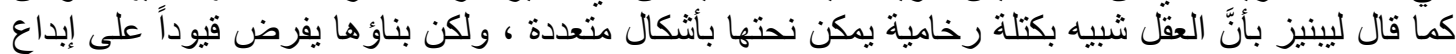

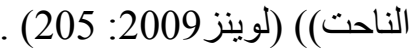

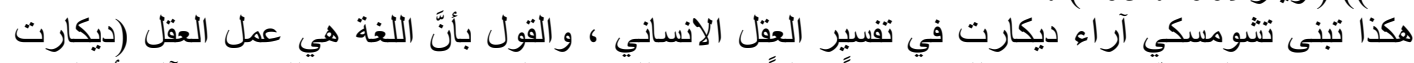

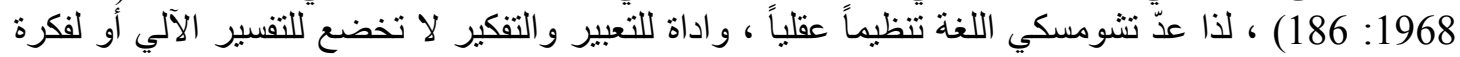
الحافز و المثير . ومن أهم المسائل التي استمدها تشومسكي من فلسفة ديكارت ما يأني : اللغة و عمل العقل. فكرة الفطرة اللغوية. وسنتناول الحديث عن هاتين المسألتين و على النحو الاتي:

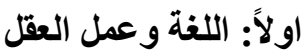

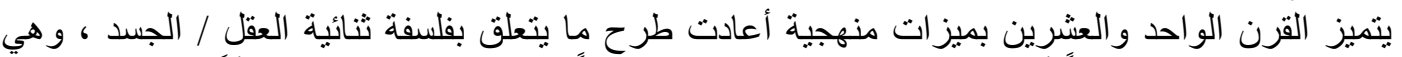

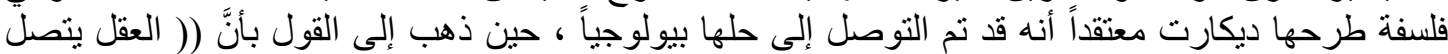
بالجسد في الغدة الصنوبرية)) (ديكارت 1968: 216) ، فما إن بدأت فلسفة العقل تظهر بوضول الكرح حتى شقت

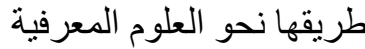

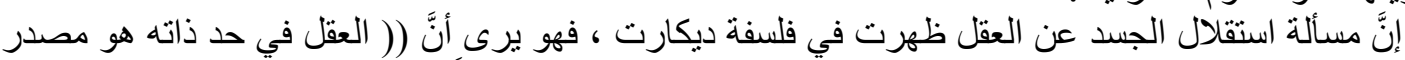

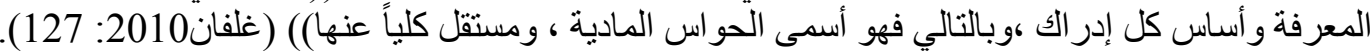

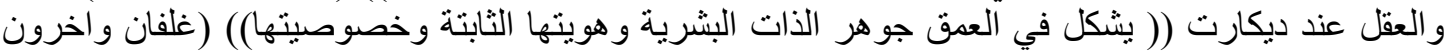

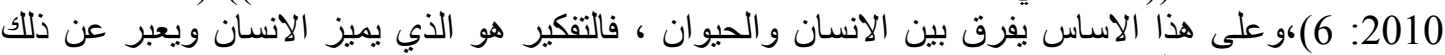

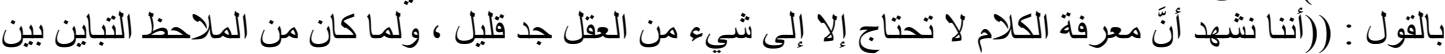

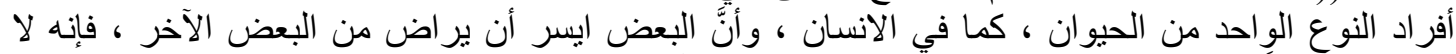

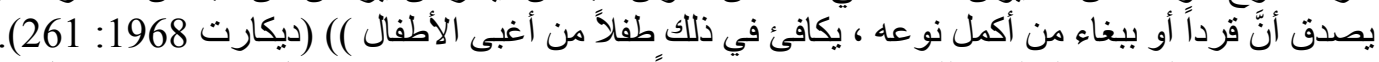

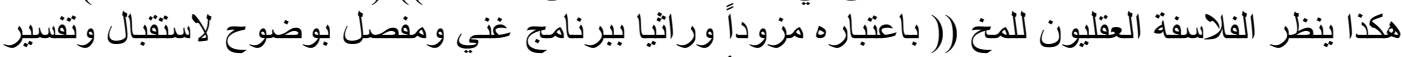

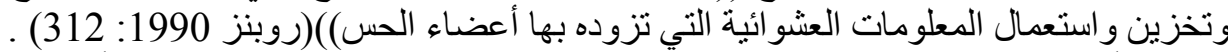

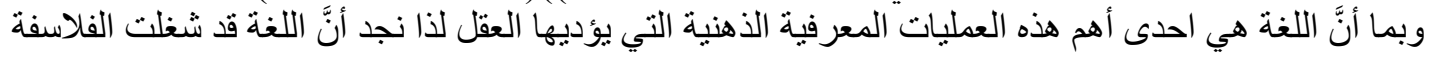
العقليين.

ينطلق ديكارت في تعامله مع اللغة من إثكالية تتعلق بطبيعة العلاقة بين اللغة و الفكر ، وهي إنكالية قديمة

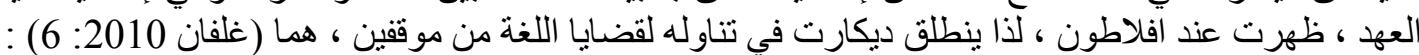




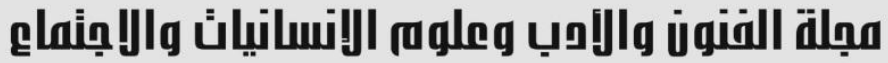

Journal of Arts, Literature, Humanities and Social Sciences www.jalhss.com

لـــــ من حيث إنَّها وسيلة غير دقيقة ، لا تصلح للتفلسف ، لأنها غير قادرة على الوصول بالإنسان إلى التعبير

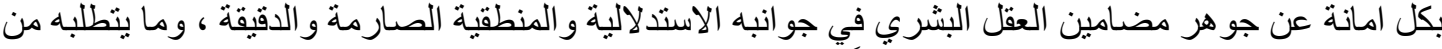

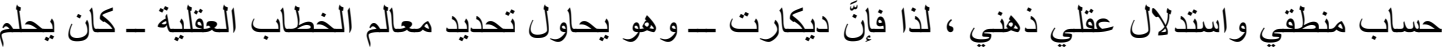

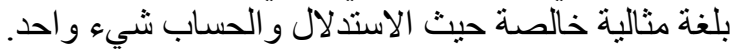

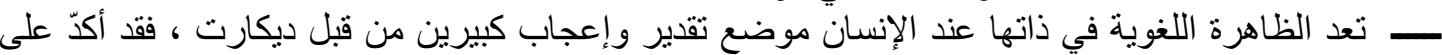

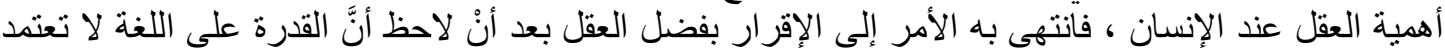

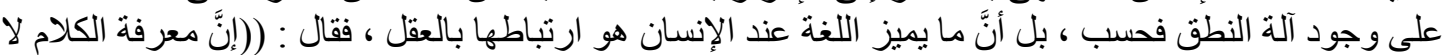

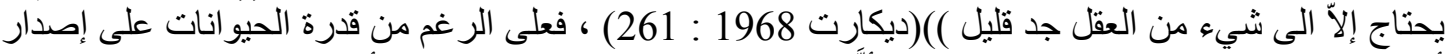

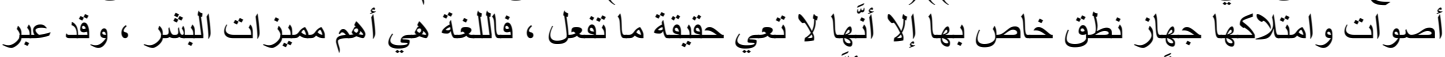

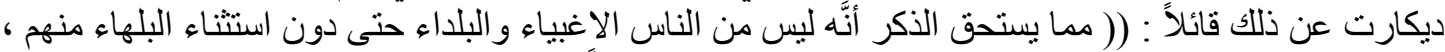

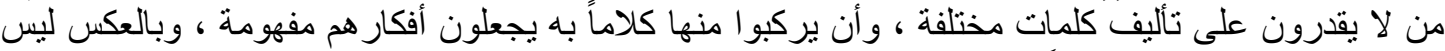

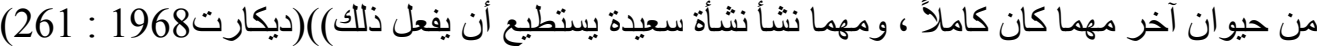

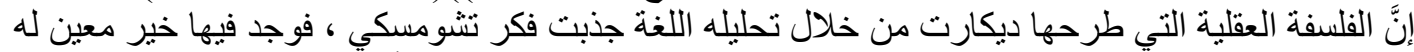

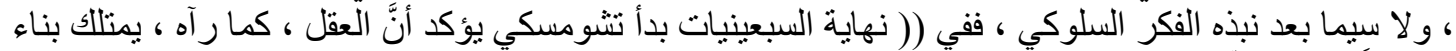

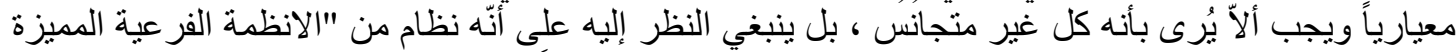

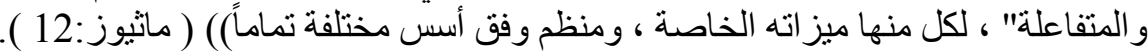

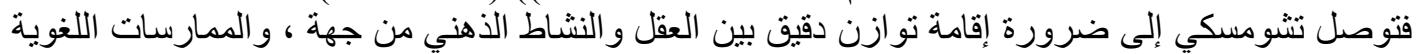

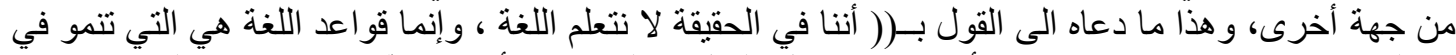

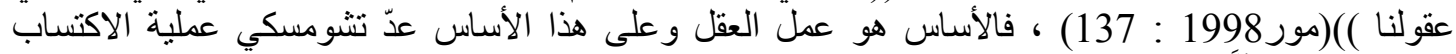

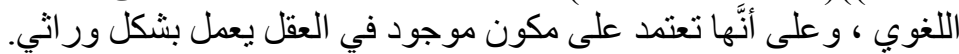

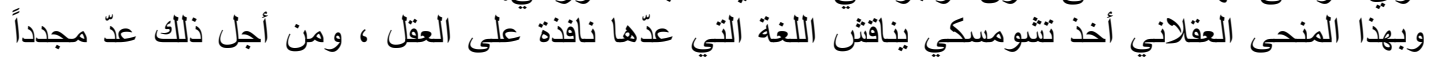

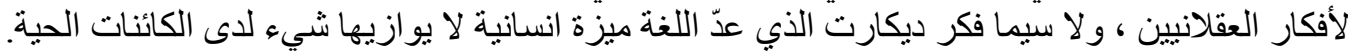

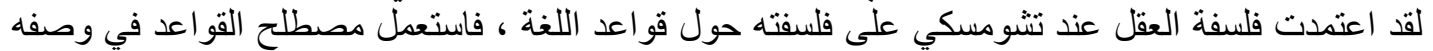

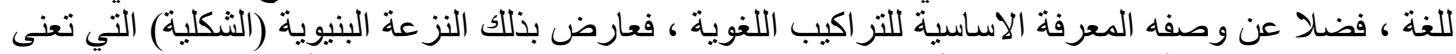

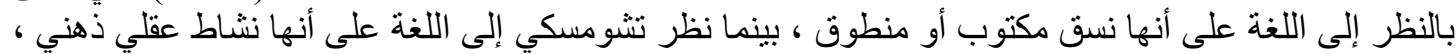

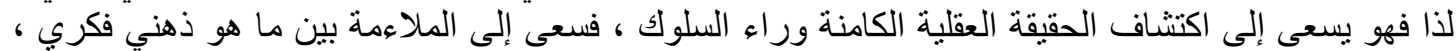
وما هو لغوي.

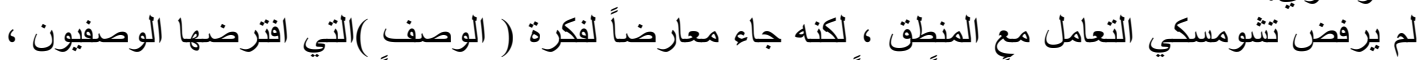

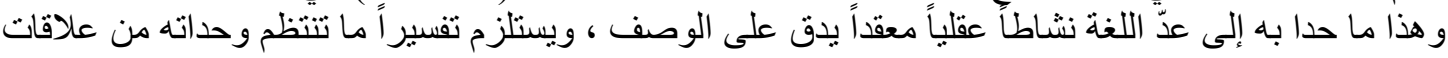
متشابكة.

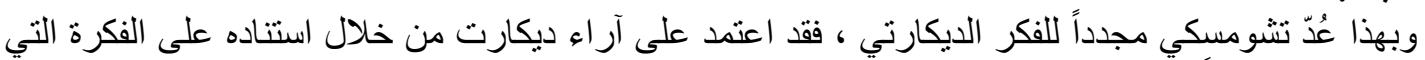

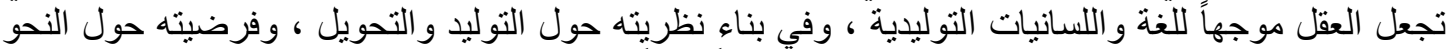

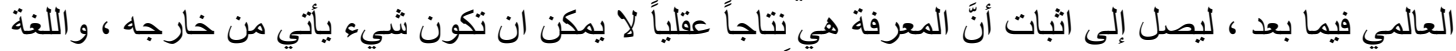
هي الوجهة المنطوقة لعمل العقل ، و هذا يعد إخلاصياً للرؤية العقلية الديكارتية.

فكرة الفطرة اللغوية

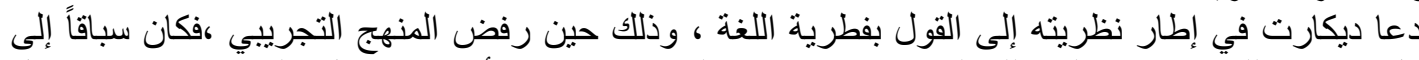

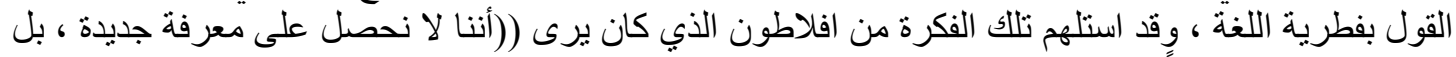

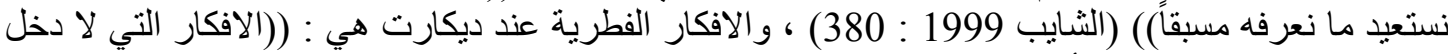

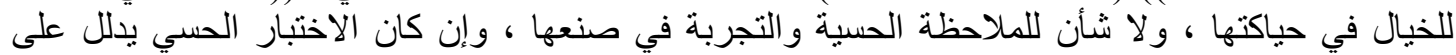

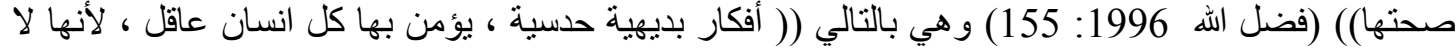
تثير أدنى شك في صحتها)) (فضل الله 1996: 155) ، و وقد ضمن كتابه " تأملات ميتافيزيقية في الفلسفة 


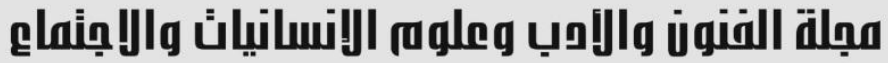

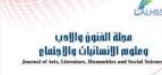

Journal of Arts, Literature, Humanities and Social Sciences www.jalhss.com

الاولى" بعضا من ذللك ، فقال : ( ( ينبغي لنا كي نقيم العلوم على قو اعد ثابته ، أن نرفض كل آر ائنا القديمة ، مرة

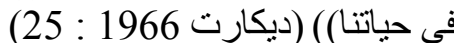

ثم نر اه يرفض فكرة معطيات الحواس ، فهو يرى أنّ من أهم ما يجب التشكيك به ، و وتحرير العقل وتطهيره

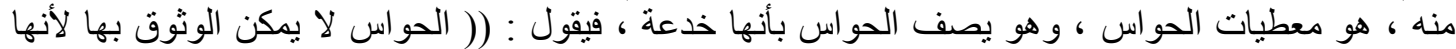

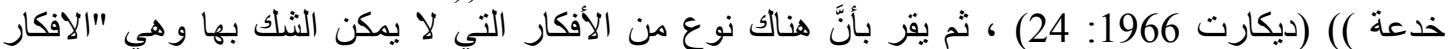

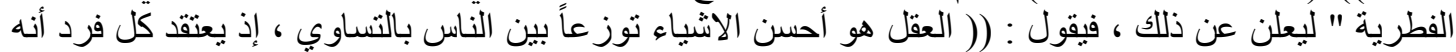

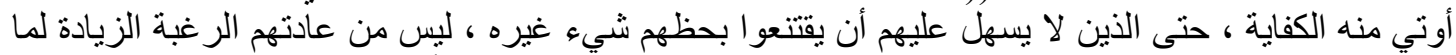

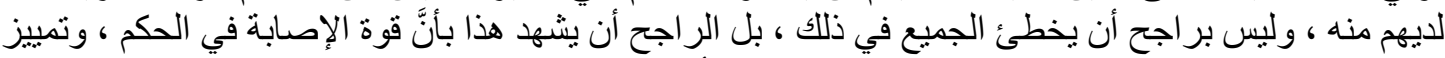

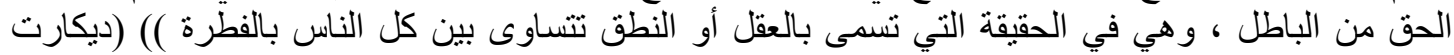

. 1966 إنَّ العقل حسب ديكارت قد وزع بين الناس بالفطرة ، لم يوجد فار غاً لينتظر ما تجود به التجربة عليه ، بل هو مزوداً بجملة من الأفكار. وعلى هذا (( فالأفكار الفطرية بالنسبة لديكارت ، هي تللك التي تنشأ عن ملكة التفكير وليس عن الأشياء

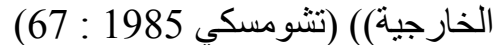

أمّا عن ماهية هذه الافكار الفطرية ومصدرها ،فإنَّ ديكارت ينظر إليها من وجهتين (فضل الله 1996 : 155) :

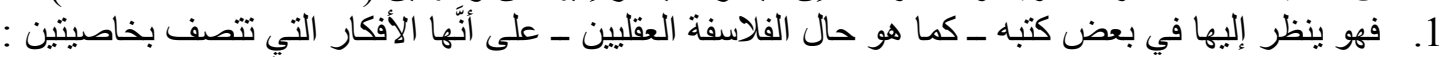

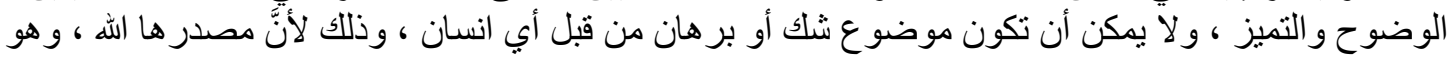

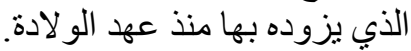

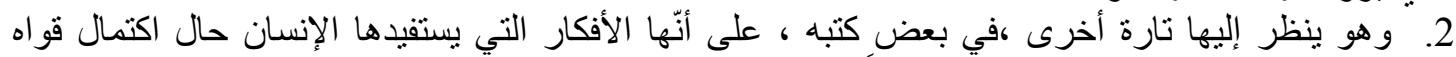

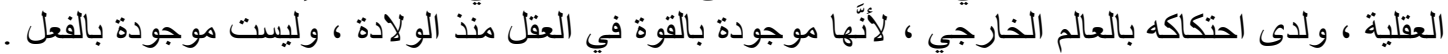

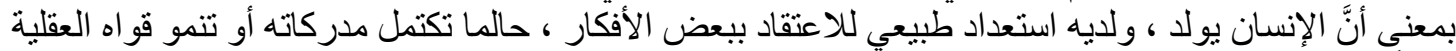

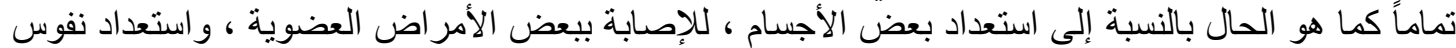
البعض للبذل و العطاء.

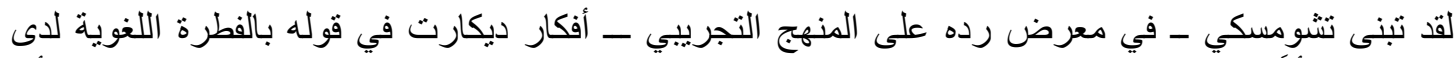

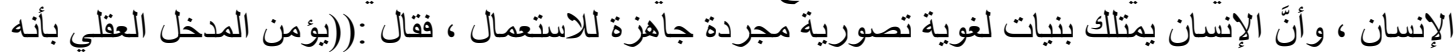

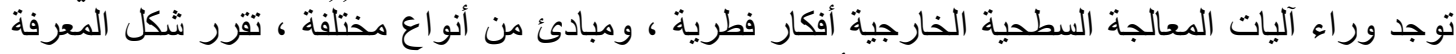

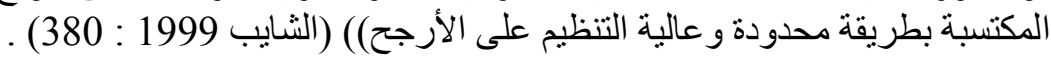

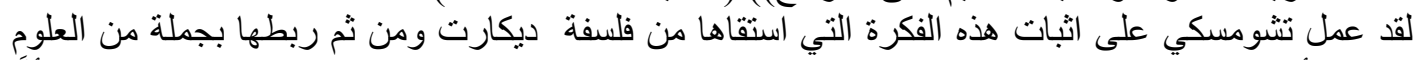

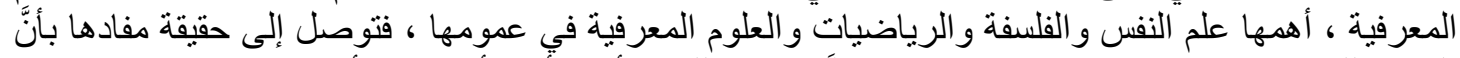

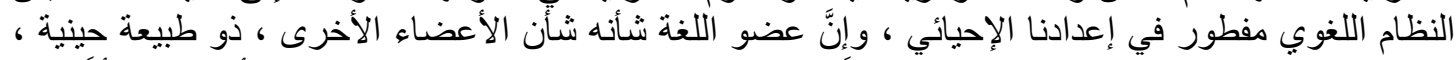

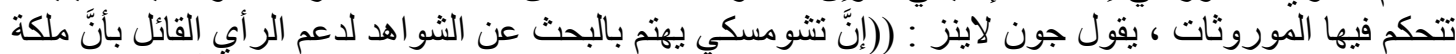

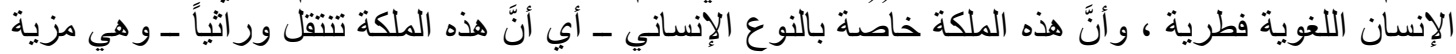

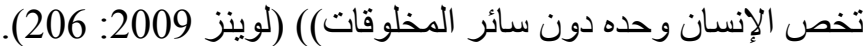

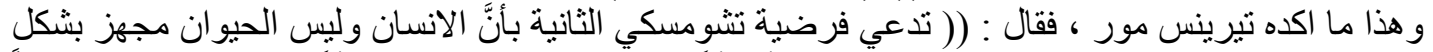

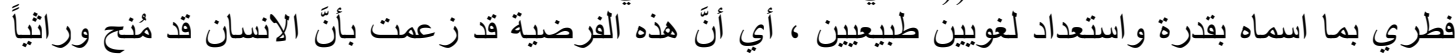

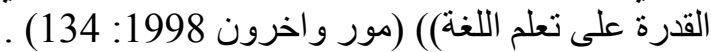

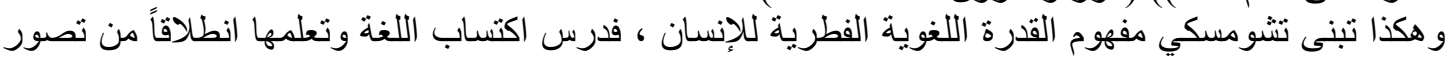

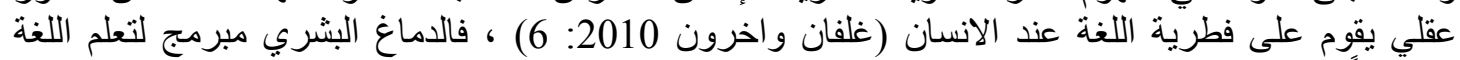

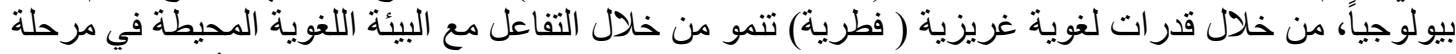

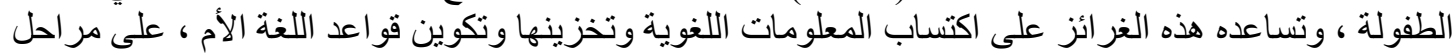

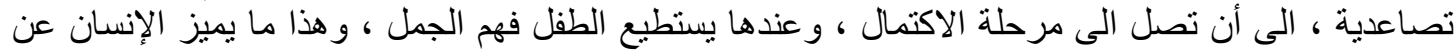




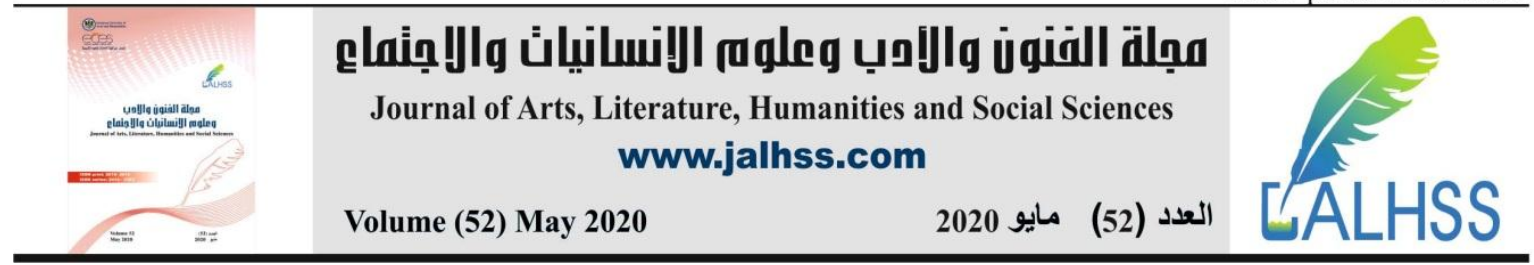

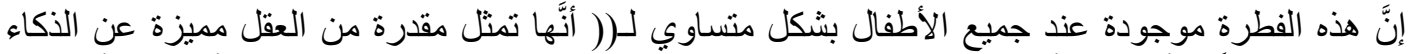

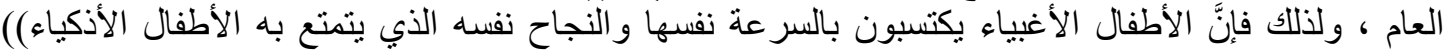

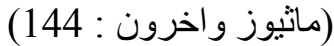
و السبب في ذللك يعود إلى كون هذه الملكة ، هي ملكة فطرية ، يجب أنْ تكون ضمن البناء الور اثي للإنسان .

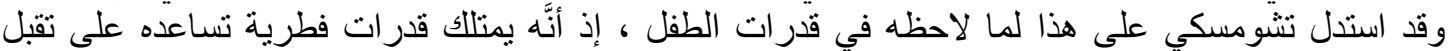

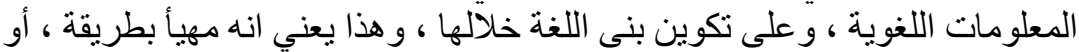

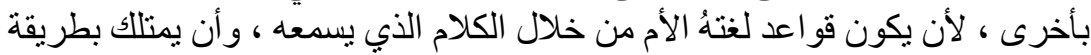

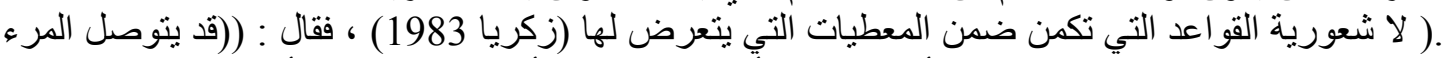
الى نظام للقو اعد عن طريق الفطرة ، أو الحدس ، أو الملاحظات الأسلوبية الجزئية ، أو الاعتيات : الاعتماد على خبرة

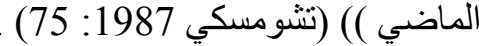

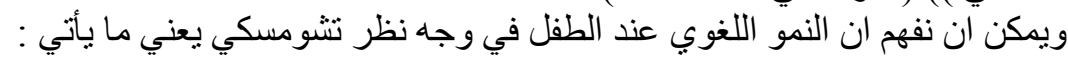

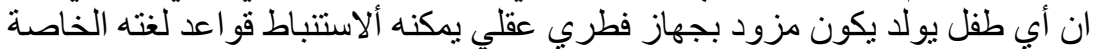
باه من استعمال المحيط الآني للغة لغنة r ان أي طفل يكون مزود بمادة لغوي عامة وكلية ، و عملة المتميز هو تحديد خصاص

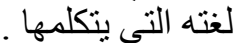

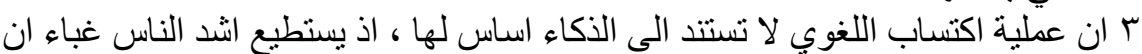

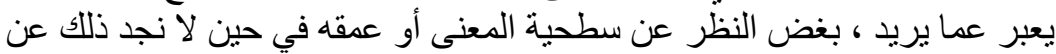

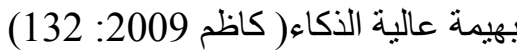

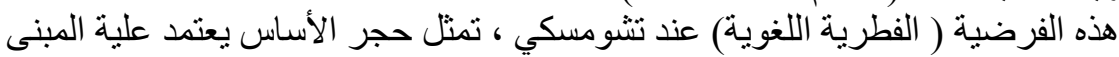

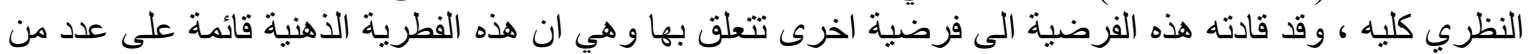

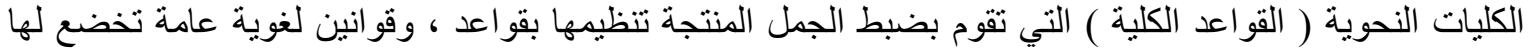

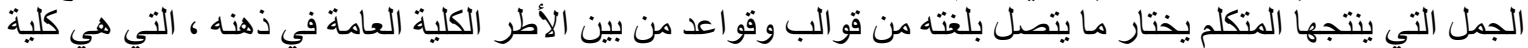

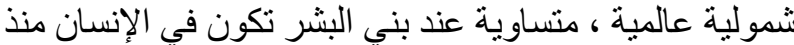

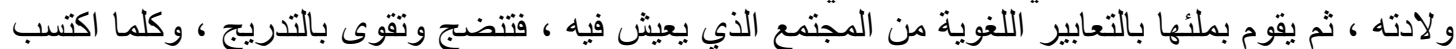

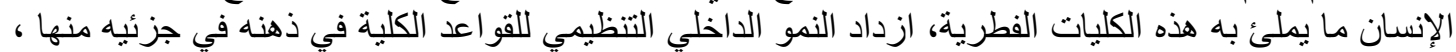

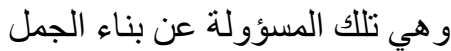

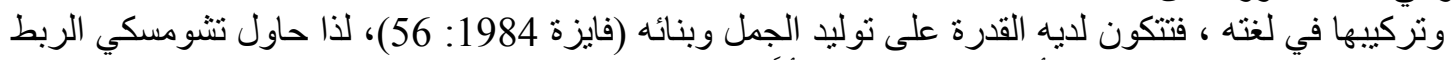

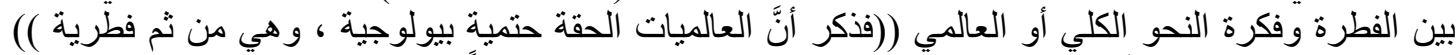

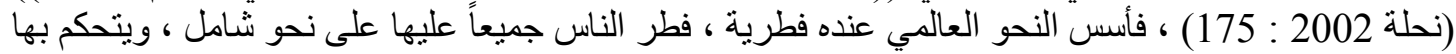

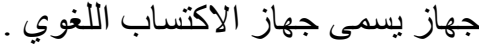
لقد غدا الموضو جهاز الاكحوري في الدراسة اللغوية عن تشومسكي هو ( المعرفة ) التي يمتلكها ابناء اللغة ، التي التي

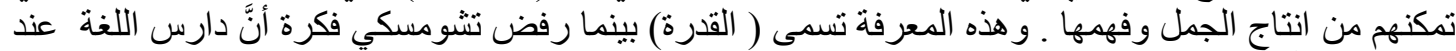

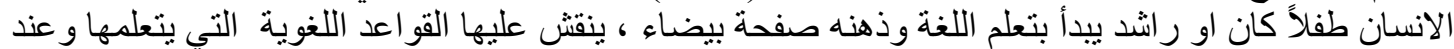

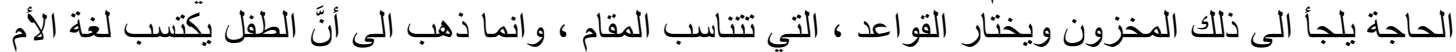

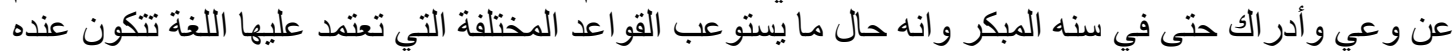

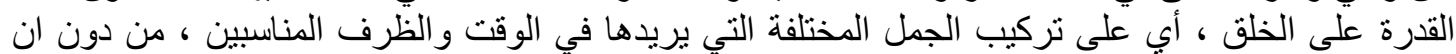

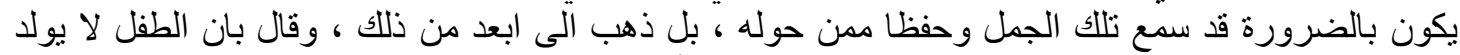

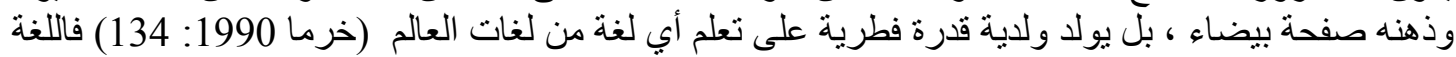

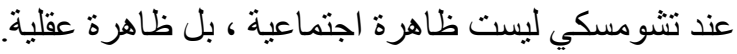

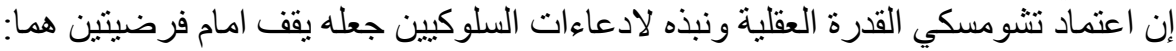

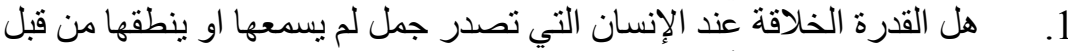

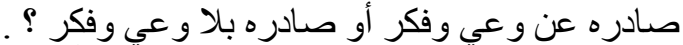

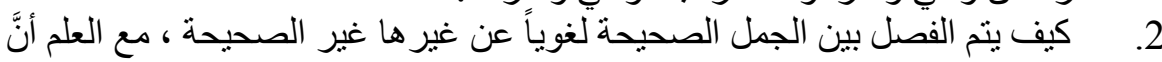




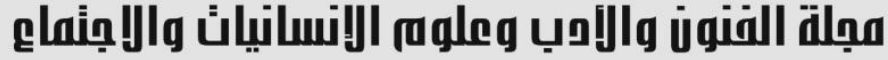

Journal of Arts, Literature, Humanities and Social Sciences www.jalhss.com

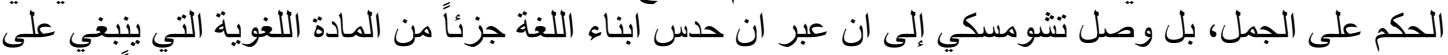

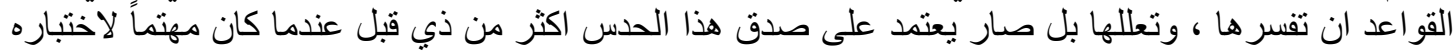

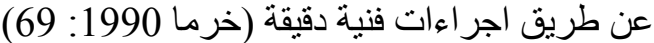
و تشومسكي هنا يناقض البنيويين الذين يعتمدون على ما يسمى بـ بـ المدونة الكلامية ) التي تجمع عن طرق

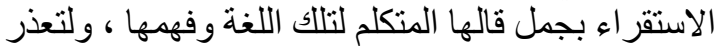

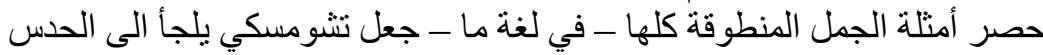
اللغوي ويعتمده (زكريا 1983 1920 262). و هذا القول بالحدس يعود بنا الى ديكارت ، بل إلى إلى افلاطون الذي اتخذ من حوار سقر اط مع العبد الذي يتعلم

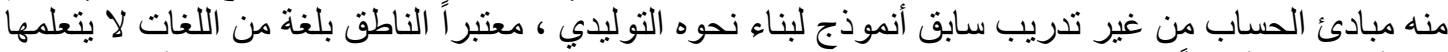

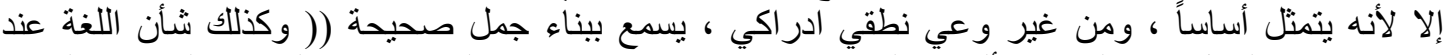

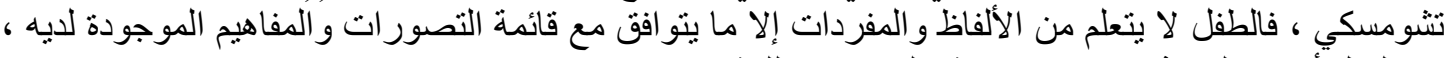

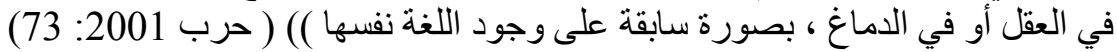

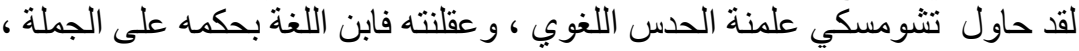

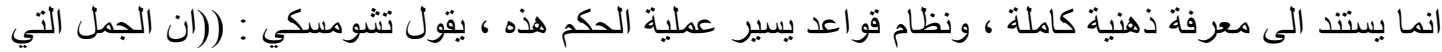

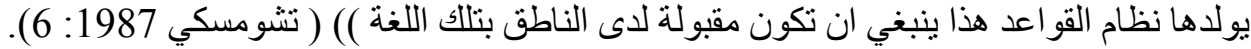

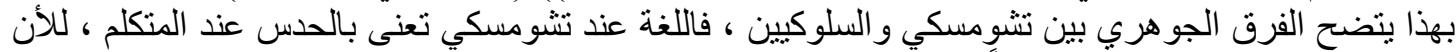

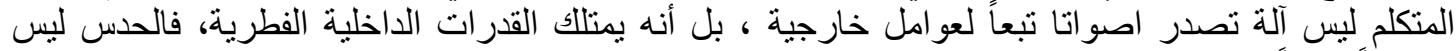

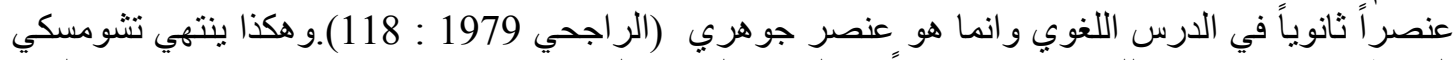

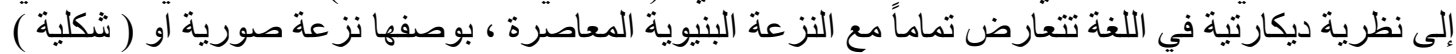

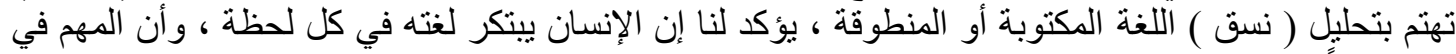

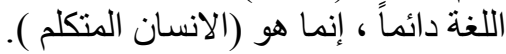

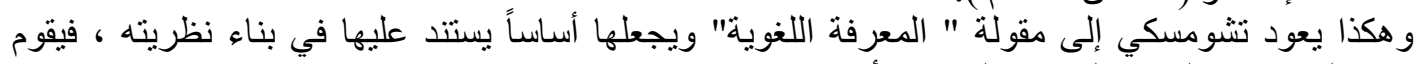

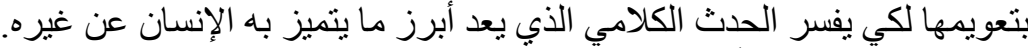

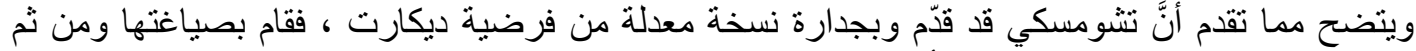
تحليل اللغة بموجبها ، وقد اقر صر احة بأثر ديكارت في تفكيره اللغوي وبناء نظريته اللغوية.

الخاتمة والنتائج

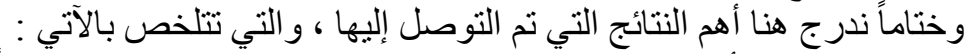

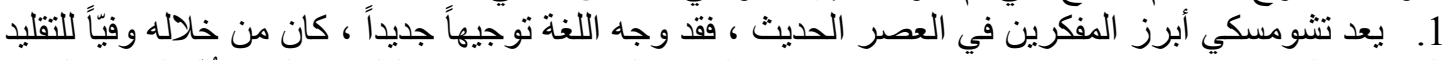
العقلاني الديكارتي ، فلا يمكن فهم نظريته من دون العودة إلى فكر ديكارت الفلى فئي الذي عُدّ الموجه لفكره اللغوي عامة. 2. تحمل فلسفة تشومسكي نزعة عقلية في البحث عن أساس معرفي ، وفلسفته هي استمرار للفلسفة التحليلية التي تضع اللغة في مركز التحليل الفلسفي. 3. رفض تشومسكي التحليل السلوكي للغة ، وِ أقرّ بوجود تأثير فطري داخل عقل الإنسان يساعده على فهم /

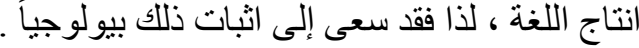




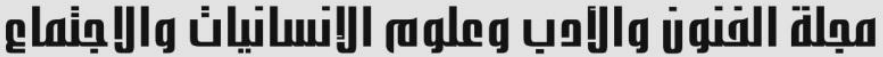

Journal of Arts, Literature, Humanities and Social Sciences www.jalhss.com

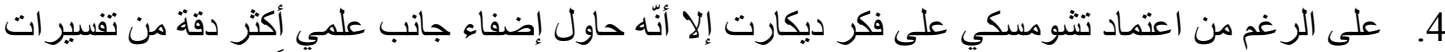

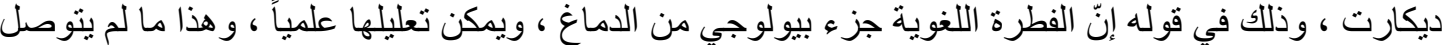
إليه ديكارت

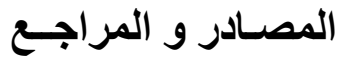

1. تشومسكي ـ ن(1987) البنى النحوية،11 ، بغداد :دار الثؤون الثقافية

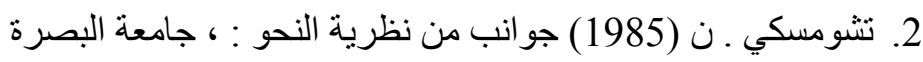

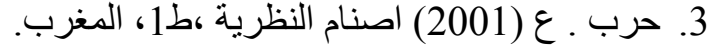

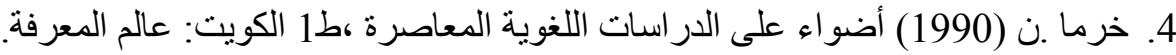

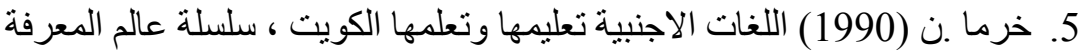

6. روبنز ـ هـ (1990) موجز تاريخ علم اللغة عند الغرب ،الكويت ، عالثات عالم المعرفة

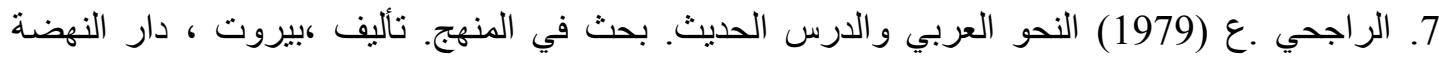
العربية. - مات.

8. ديكارت ـ ـ (ب.ت) تأملات ميتافيزيقية في الفلسفة الاولى ، بيروت ـ لبنان

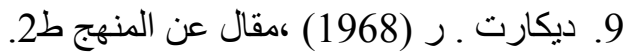

10. ركريا. م (1983) الالسنية ( علم اللغة الحديث) المبادئ و الاعلام ،ط2، بيروت : المؤسسة الجامعية

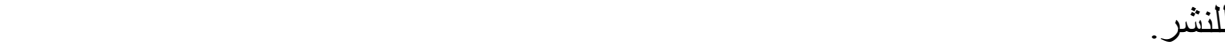

11. السيد ـ ص (1989) تشومسكي فكره اللغوي وآراء النقاد فيه، الإسكندرية: دار المعرفة .

12.

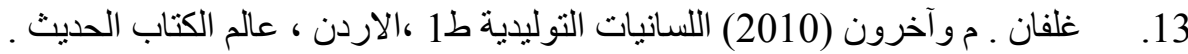

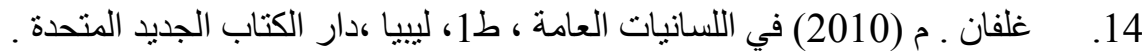

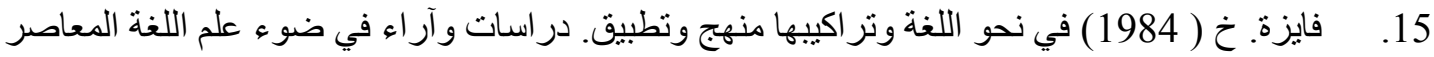

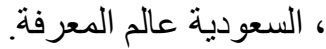

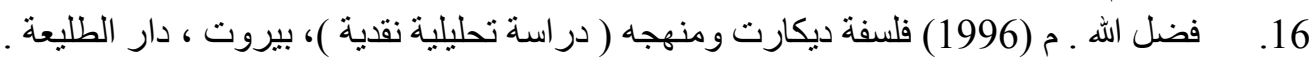

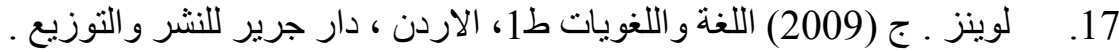

18.

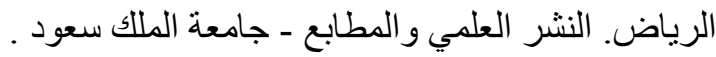

19. 19 مور .ت (1998) فهم اللغة : نحو علم لغة لما بعد مرحلة تشومسكي : ط1، بغداد دار الثؤون الثقافية.

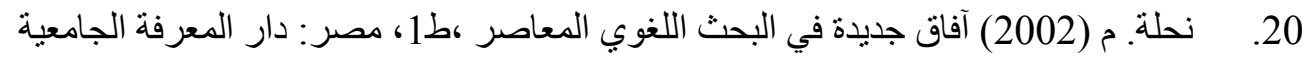




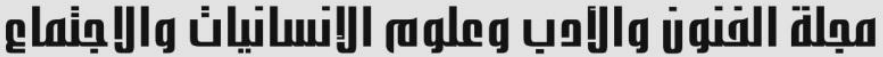

Journal of Arts, Literature, Humanities and Social Sciences

www.jalhss.com

\section{References}

1. Chomsky. N (1987) Grammatical Structures, 1st edition, Baghdad: House of Cultural Affairs.

2. Chomsky. N (1985) Aspects of grammar theory: University of Basra.

3. War . P (2001) Idols of Theory, 1st ed, Morocco.

4. Kharma N (1990) Spotlight on Contemporary Linguistic Studies, 1st edition Kuwait: The World of Knowledge.

5. Kharma N (1990) Foreign Languages Teaching and Learning Kuwait, Knowledge World Series.

6. Rubens. E (1990) A summary of the history of linguistics in the West, Kuwait, the world of knowledge.

7. Al-Rajhi. A. (1979) Arabic Grammar and Modern Lesson. Research the curriculum. Written by, Beirut, Arab Renaissance House

8. Descartes. RPT metaphysical reflections on the first philosophy, Beirut Lebanon.

9. Descartes. R. (1968), an essay on the curriculum, 2nd edition.

10. Zakaria. M (1983), Al-Sunni (Modern Linguistics) Principles and Information, 2nd edition, Beirut: The University Publishing Foundation.

11. Al-Sayed . P. (1989) Chomsky Linguistic Thought and Critics' Opinions in it, Alexandria: Dar Al-Maarefa.

12. Al Shayeb. P (1999) Lectures in Linguistics, I 1'Amman - Jordan, Ministry of Culture.

13. Galvan. M et al. (2010) Obstetric Linguistics I 1, Jordan, Modern Book Scientist

14. Galvan. M. (2010) in General Linguistics, 1st edition, Libya, United New Book House.

15. Fayza. KH (1984) Towards Language and Its Compositions: Methodology and Application. Studies and opinions in the light of contemporary linguistics, Saudi Arabia's world of knowledge.

16. The grace of God. M. (1996) Descartes' Philosophy and Method (Critical Analytical Study), Beirut, Dar Al-Tale'ah.

17. Lewins. C (2009) Language and Linguistics, 1st floor, Jordan, Dar Jarir for Publishing and Distribution.

18. Matthews. E (N.D.) Language is a mental ability, offering Chomsky (within the Language Encyclopedia), Saudi Arabia - Riyadh. Scientific publishing and printing - King Saud University.

19. Moore T. (1998) Understanding Language: Towards Post-Chomsky Linguistics: 1st Floor, Baghdad, House of Cultural Affairs.

20. Nahla . M. (2002) New Perspectives in Contemporary Linguistic Research, 1st edition, Egypt: Dar Al-Maarefa Al-Jami’ia. 